\title{
Fostering intuitive knowledge of multivariable calculus concepts using a collaborative augmented reality application
}

DOI: https://doi.org/10.17230/9789587207002lch3

Juan Sebastián Vega, Agustín Nieto, Camilo Córdoba,
Guillermo Alejandro Gil, Helmuth Trefftz, Pedro Vicente Esteban

\section{Introduction}

Education has been constantly evolving along with many other human processes and activities. Computers are now key elements within the learning process that help educators and students attain academic goals. Whilst some tools are developed for strengthening soft skills (Holzapfel, 2018; Manyika et al., 2017), others assist students in different learning areas. Universities as well as primary and secondary schools have identified that certain technologies are useful to support the teaching-learning process (Rickel, 2001; van den Berk, 2015).

Mathematical concepts are, in some cases, abstract and not easy to understand and visualize. Calculus, and multivariable calculus in particular, is an example of such a complex area (Orozco et al., 2006). Trying to draw a 3D surface on a 2D plane, such as a whiteboard or a piece of paper, is, in many cases, difficult and hard to achieve as well as timeconsuming and not efficient. There are some tools available in the market that help educators teach some calculus concepts, such as GeoGebra which is a powerful and useful tool but is still a 2D representation of a 3D surface (Di Serio et al., 2013; Freina \& Ott, 2015; Ibáñez \& DelgadoKloos, 2018; Ibáñez et al., 2020; Volkow \& Howland, 2018).

Virtual and augmented reality technologies (VR and AR, respectively) are having a huge impact on many aspects of human life, one of them being education (Azuma et al., 2001; Burdea \& Coiffet, 2003). They provide environments that help students overcome some of the problems mentioned above (Giraldo et al., 2007; Pan et al., 2006; Velev \& Zlateva, 2017) and increase motivation in both educators and students (Jensen 
\& Konradsen, 2018). There are different technologies that support VR experiences: some of them track only users' head movements, while others track their whole-body movements. The type of tracking seems to have an impact on the students' learning process, as suggested by Alhalabi (2016).

This study describes the development of an AR-based tool that helps calculus teachers and students reach a desired academic goal, aiming to achieve a high level of acceptance of, satisfaction with, and motivation in the teaching-learning process.

This article is organized in the following sections: Related Work introduces the state of the art of the use of technologies, mainly AR, within the educational world; Description of the AR Application and its Validation describes the usage and development of the application and how it was validated. The section Results presents the impact of the application among the selected group of students, through statistical calculations. The last section of this article is Conclusions and Future Work which contains the outcome of this research and how it is going to move forward.

\section{Related Work}

When students approach advanced studies in Science or Engineering, they have a large corpus of nonformal (intuitive) knowledge, acquired from their interaction with the natural world (Chudnoff, 2013). In some cases, this intuitive knowledge must be unlearned; for instance, when the formal knowledge to be acquired is counter-intuitive, such as the quantum phenomena at the sub-atomic level. In most cases, creating associations between the previously acquired intuitive knowledge and the concept to be studied helps students approach the learning process and acquire the necessary ulterior formalization. In the area of Physics, a study (Sherin, 2006) describes how the previous intuitive knowledge that physics students have acquired helps them by providing context to the interpretation of the physics problems presented to them.

There has been a revival of interest in VR and AR technologies, particularly for educational purposes (Chen et al., 2017). Multiple factors explain this revival: one of them is the availability of more powerful yet less expensive hardware that can render quite complex scenes and 3D objects; another is their business potential: various sources predict the VR/ AR market to grow to 161.1 billion US dollars by 2025 (GlobeNewswire, 
2020). One of the most appealing uses of VR/AR is in education and training: VR/AR technologies can be used to teach procedures and concepts, increasing the users' engagement and immersion, while reducing risks and costs.

Augmented reality is being used more and more in educational settings. Ibáñez \& Delgado-Kloos (2018) found 28 studies using AR to support the learning of Science, Technology, Engineering and Math (STEM) concepts. Most of the studies aimed at allowing students to learn through exploration or simulation activities. The reported studies prove that "augmented reality technology fosters positive affective states of students, such as motivation, engagement, and attitudes toward STEM subjects that have proved to be effective in promoting learning benefits" (p. 12). The study by Martín-Gutiérrez et al. (2015) shows that AR can be used effectively to promote autonomous learning in higher education.

Our work differs from the above in that we created and validated an AR application specifically to develop intuitive knowledge of multivariable calculus in first-year engineering students. The specifics of the study are described in the following section.

\section{Description of the AR Application and its Validation}

To provide students with a 3D visualization of the surfaces and planes, a collaborative AR application was created using Vuforia for visualization and Photon for collaboration. The application allowed the instructor to create a function of the form

$$
z=f(x, y)
$$

After the instructor entered the function, all participants were able to observe, through their cell phones, the corresponding 3D surface rendered on top of a printed pattern, which was typically placed on top of a table in the middle of the classroom (see Figure 1). 
Figure 1. Student holds his cellphone in his hand, pointing at the pattern. Note the 3D surface that is rendered on the cellphone

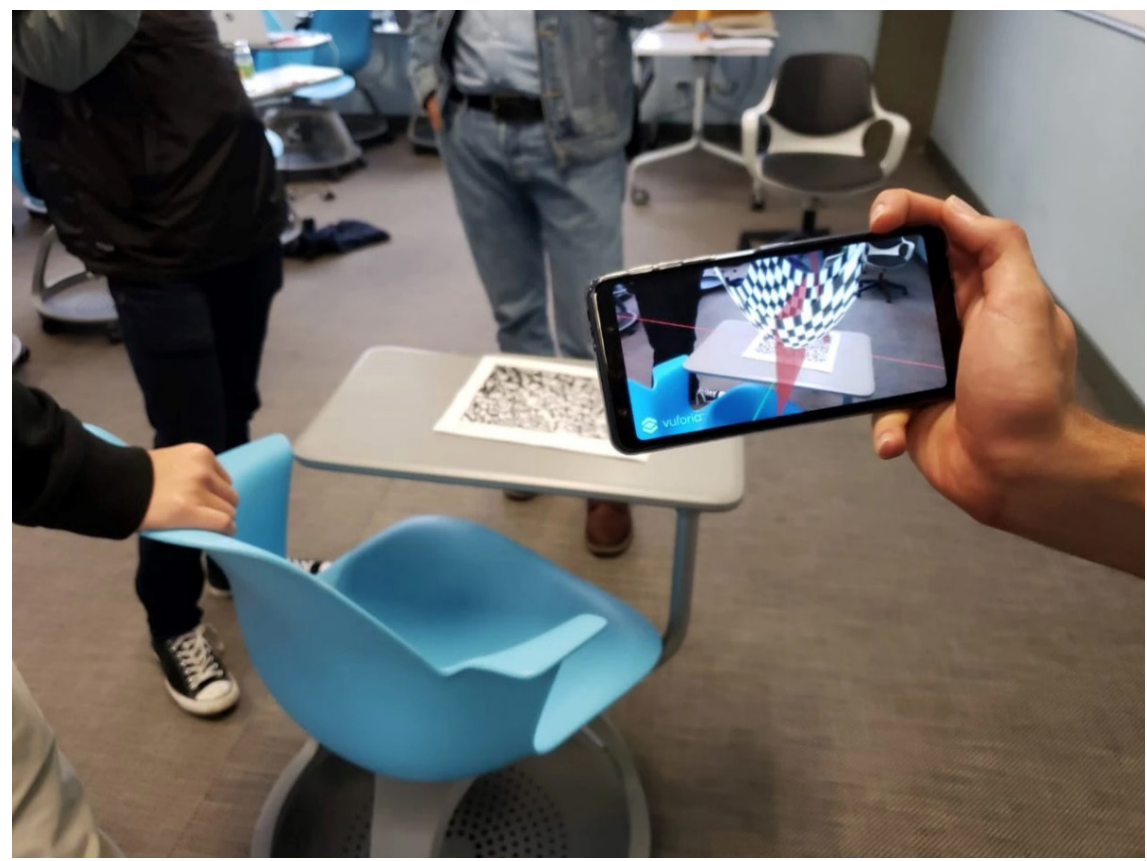

Source: Prepared by the authors

Development of the AR application

The application provides support for two distinct types of user: instructors and students. Instructors are responsible for creating the "room", i.e., the shared virtual space in which the collaboration messages are broadcast. After the room is created, instructors enter the equation of the surface to be visualized. The instructor can restrict the domain of the $\mathrm{x}$ and $\mathrm{y}$ variables, and scale the surface to be larger or smaller, to create a better visualization. After joining the virtual room, students visualize the surface that the instructor has added. Both the instructor and the students can point at any arbitrary area in the shared surface, touching their cell phones with their fingers. When a user $\mathrm{X}$ points at an arbitrary area, other users visualize a "ray" originating at X's cell phone and intersecting with the surface. This allows every participant to see where participant $\mathrm{X}$ 
is pointing at. It is important to mention that this functionality allows for a remote usage of the application where users could be at different physical locations.

To facilitate the definition of the function to be visualized, a parser was created. The parser recognizes functions including polynomials and trigonometric functions. After being parsed, the function is evaluated at various points of the $\mathrm{XY}$ plane, and a polygon mesh, representing the surface, is created (see Figure 2).

Figure 2. 3D rendering on the $\mathrm{AR}$ application of equation $z=x^{2}-y^{2}$

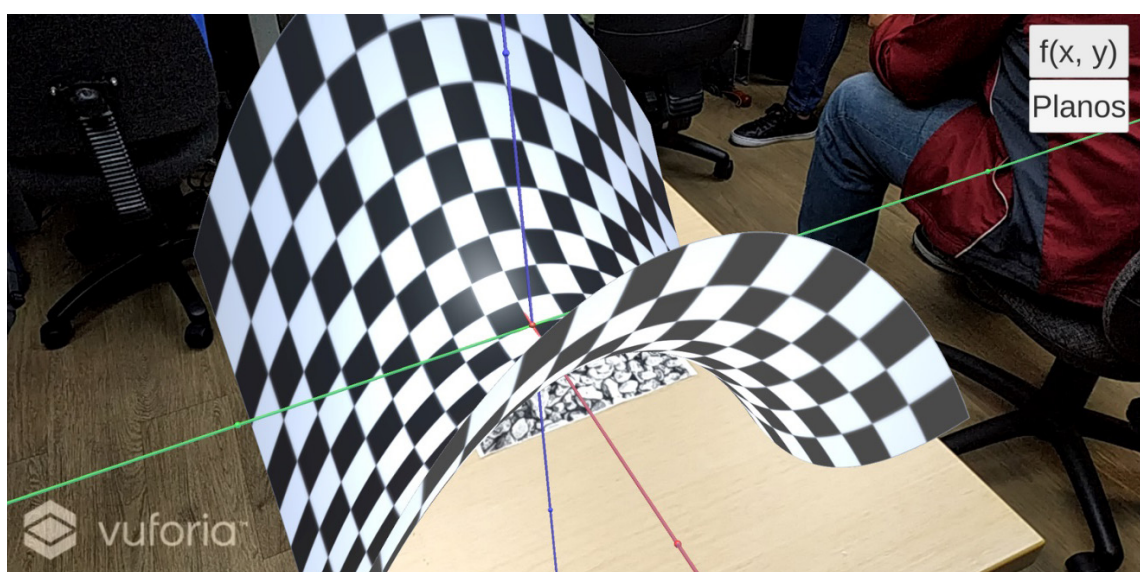

Source: Prepared by the authors

The AR application, was built using parts of several existing applications: Vuforia (https://www.ptc.com/en/products/vuforia), Photon (https://www.photonengine.com/) and Unity (https://unity.com/) frameworks. It was run on the students' Android mid-range cell phones without any major inconvenience. The limitation on the number of users permitted by the free version of Photon was not a problem, since the virtual rooms were shared by no more than 10 simultaneous users. 


\section{Pedagogical use of the AR application}

After the initial setup, the instructor asked the students to observe the surface from various points of view. In the case of the hyperbolic paraboloid depicted in Figure 2, the instructor asked students to describe the parabolic curves forming the surface when observed from the $\mathrm{X}$ axis, shown in red (opening up), as opposed to the parabolic curves forming when the surface is observed from the $\mathrm{Y}$ axis, shown in green (opening down). The instructor asked students also to cast rays that moved away from the midpoint of the surface $(x=0$ and $y=0)$ in the direction of the $\mathrm{X}$ axis, and showed them that they would be going "up," as opposed to moving away from the mid-point in the direction of the $\mathrm{Y}$ axis, in which case they would be going "down." The instructor used this activity to show students the intuitive concept of partial (and directional) derivatives. Students answered questions asked by the instructor to reach consensus on the explanations. Similar guided experiences were used to introduce the concept that the intersection between a 3D surface and a plane, if it exists, forms one or several curves in space (see Figure 3).

Figure 3. Intersection of the function $\mathrm{z}=\sin (\mathrm{x})+\cos (\mathrm{y})$ and a plane that is parallel to the $\mathrm{XY}$ plane

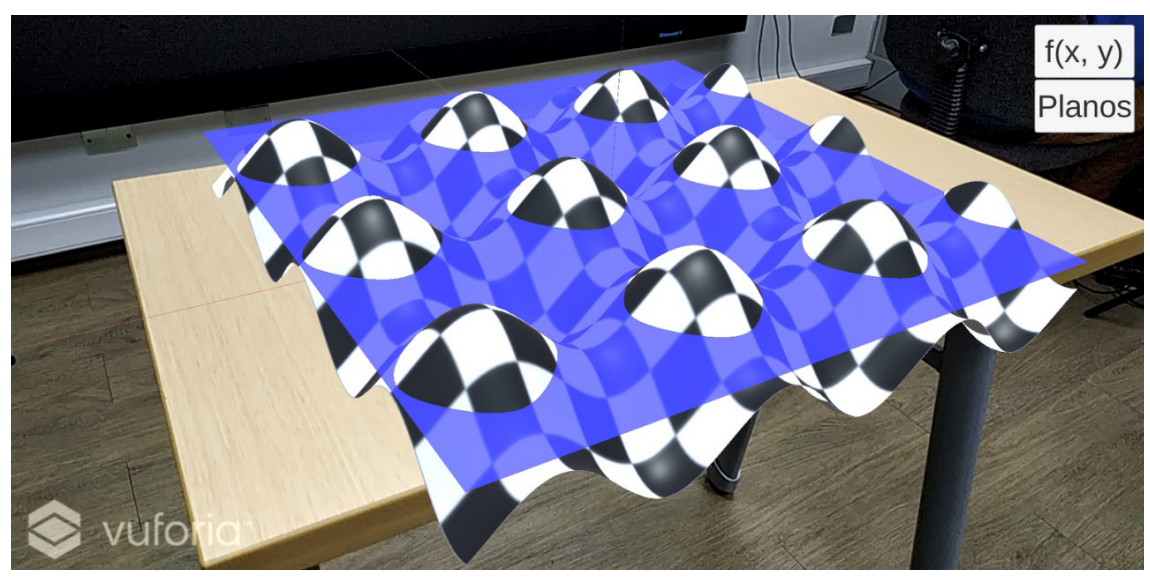

Source: Prepared by the authors 
We believe that it is the self-reflection process, undertaken by the students during the collaborative session, and supported by the technology, that enhances their understanding of the topic at hand. It is, therefore, not the technology by itself, but in combination with an appropriate pedagogical approach that is pedagogically effective.

\section{Pedagogical validation of the AR application}

Users with no knowledge in calculus (first-year students) were asked to complete pre and post-test evaluations. Pre and post-test evaluations contained the same questions but were presented at different times and aimed to measure the knowledge acquired during the experience. The questionnaire was designed by the researchers because of the need to address very specific knowledge of multivariable calculus in several questions, while, in others, addressing specific motivation questions.

Questions in bold are the ones considered for measuring the knowledge variation (delta) (see Table 1).

Students also completed questionnaires that aimed at evaluating their subjective perceptions of the experience. The questionnaires are built as a set of affirmations and students choose options from a Likert scale. The following topics were explored in the questionnaires:

- Personal satisfaction

- Relevance of the tool

- Personal perception

- Tool's usability

- Learning

- Motivation

- Attention in classes (during the lecture)

- Attention in classes (during the activity)

\section{Results}

This section presents the results of the students' assessment, both on the level of knowledge before and after the intervention and on the students' perceptions of the application. 
Table 1. Questionnaires that students completed before and after the pedagogical intervention

In the following questions, you will read about a series of situations regarding calculus and surface calculus. Answer freely. Answers will be used for research purposes only.

In each case, please select at least one option and indicate any others you consider relevant.

1. Some everyday situations in which calculus is applied are:
a. For calculating volumes.
b. For finding tangent planes to a surface in each point on the surface.
c. For finding maximum and minimum points along a function or surface.
d. Other:

2. Some specific situations in which you have used calculus outside academic work are:
a. Personal work.
b. In the development of a personal hobby.
c. None.
d. Other:

3. In your surroundings (home, university, city) is there any surface that you could describe with equations or calculus functions?
a. The roofs of some building.
b. Some ornamental figures.
c. I have not seen surfaces that could be described with calculus equations.
d. Other:

4. Do you think that the study of equations through calculus can help in the understanding of concepts within other courses or modules of your course?
a. Velocity.
b. Acceleration.
c. Direction.
d. Other:

5. Studying and understanding surfaces through their equations is important because they allow me to:
a. Model my surroundings.
b. Build 3D scaled models.
c. Calculate maximum, minimum and saddle points.
d. Find directions of points within the surface.
e. Other: 
6. If you are standing on a surface, in what way could you determine, if it exists, if there is a maximum point or points on it:

a. If you observe, from all surface points, that to reach that point you have only a positive slope.

b. If you observe, from all surface points, that to reach that point you have only a negative slope.

c. If you observe, from all surface points, that to reach that point you have negative and positive slopes.

d. Other:

7. If you are standing on a surface, in what way could you determine, if it exists, if there is a minimum point or points on it:

a. If you observe, from all surface points, that to reach that point you have only a positive slope.

b. If you observe, from all surface points, that to reach that point you have only a negative slope.

c. If you observe, from all surface points, that to reach that point you have negative and positive slopes.

d. Other:

8. Could some points be maximum and minimum at the same time?

a. No. There could only be maximum points or minimum points or points that are neither of those.

b. Yes. There could be points that, when reaching them in some directions, you have positive slopes while, when reaching them from other directions, you have negative slopes.

c. All points along a surface have the same characteristics.

d. Other:

9. If you cut a surface with a plane you could get:
a. A point.
b. A curve.
c. You could not determine the shape of the figure.
d. You do not get any figure.
e. Other:

10. If you are located on a surface, how would you define the direction with higher slope?
a. You will walk along the surface and notice the direction in which you gain height.
b. You will walk along the surface and notice the direction in which you lose height.
c. You will notice the direction in which you do not lose or gain height.
d. Other: 
11. Could surfaces you see daily could be represented as mathematical equations?

a. No. There is no way of describing equations for surfaces in my surroundings.

b. Yes. You could represent a surface in my surroundings through some equations.

c. If it were possible to find equations to represent any surface, there would be more designs.

d. Other:

12. In a point within a surface, how many tangent lines could you trace?
a. Just one. Through a point in space, only one line passes.
b. Infinite, because being at a point in space you could spin the line in any direction.
c. It depends on the point you are studying.
d. Other:

13. In a point within a surface, how many tangent planes could you trace?
a. Infinite, because the plane could take many slopes.
b. Just one. A plane could only have one slope at a given surface point.
c. You cannot draw a plane tangent to a surface.
d. Other:

14. The area of a finite surface is a quantity required during the design and building of structures. How can you calculate an approximate area of any type of surface?
a. You could draw parallel lines that go through the edge of the surface so that each part of it becomes a rectangle. Then you sum those areas.
b. You can only calculate the area of plane surfaces that are made of rectangular figures.
c. You cannot know the area of any surface.
d. Other:

15. When intercepting a plane perpendicularly with the spin axis of a rectangular cylinder, you derive:
a. A point.
b. A line.
c. A circumference.
d. Other:

16. Which of these tools have you used to visualize surfaces?
a. GeoGebra ${ }^{\circledR}$
b. Wolfram Alpha ${ }^{\circledR}$
c. $\operatorname{Desmos}{ }^{\circledR}$
d. Other: 
17. Can the study and understanding of surfaces through calculus help you know your surroundings better?

a. Yes. Calculus helps me model my surroundings.

b. Yes. Calculus helps me determine important points along the surface.

c. No. It is not easy to find equations that model surfaces.

d. Other:

In a week, how much time do you spend playing video games?

Further comments:

\section{Knowledge assessment}

All students who were involved in this research were asked to participate voluntarily. Their involvement in this project had merely research intentions. They were also told that they could leave the experiment at any time if they experienced motion sickness or any other discomfort.

Twenty students took part in the AR intervention. All of them were first-year students who had no previous knowledge of multivariable calculus. All of them took the pre and post-tests. The tests were graded on a scale from 1.0 to 5.0, 1.0 being the lowest score and 5.0 the highest. The scores of the students in the pretest and post-test with the knowledge change are described in Table 2 .

Table 2. Variation in knowledge between the pre and post-tests

\begin{tabular}{|c|c|c|c|}
\hline Subject & Pretest Grade & Post-test Grade & Variation \\
\hline 1 & 2.7 & 3.5 & 0.8 \\
\hline 2 & 3.1 & 2.3 & -0.8 \\
\hline 3 & 2.7 & 2.7 & 0 \\
\hline 4 & 2.3 & 3.8 & 1.5 \\
\hline 5 & 1.9 & 3.1 & 1.2 \\
\hline 6 & 3.5 & 2.7 & -0.8 \\
\hline 7 & 1.5 & 3.5 & 2.0 \\
\hline 8 & 3.1 & 3.1 & 0 \\
\hline 9 & 1.9 & 3.8 & 1.9 \\
\hline 10 & 2.3 & 3.5 & 1.2 \\
\hline
\end{tabular}




\begin{tabular}{|c|c|c|c|}
\hline 11 & 2.3 & 3.1 & 0.8 \\
\hline 12 & 2.3 & 3.1 & 0.8 \\
\hline 13 & 1.5 & 2.7 & 1.2 \\
\hline 14 & 2.7 & 3.1 & 0.4 \\
\hline 15 & 2.3 & 2.3 & 0 \\
\hline 16 & 1.9 & 1.9 & 0 \\
\hline 17 & 2.7 & 3.1 & 0.4 \\
\hline 18 & 1.5 & 2.7 & 1.2 \\
\hline 19 & 1.9 & 2.7 & 0.8 \\
\hline 20 & 3.1 & 3.1 & 0 \\
\hline
\end{tabular}

Source: Prepared by the authors

An ANOVA test showed that the means are significantly different with a $p$ value $<0.05(p=0.0007)$, suggesting that means of the populations (pretest and post-test) are statistically significant. Additionally, a Tukey test was carried out to find which means were significantly different. As shown in Figure 4, the second group, in red, which is the post-test group, has a significantly higher mean than the first group.

Figure 4. Results of the Tukey Test.

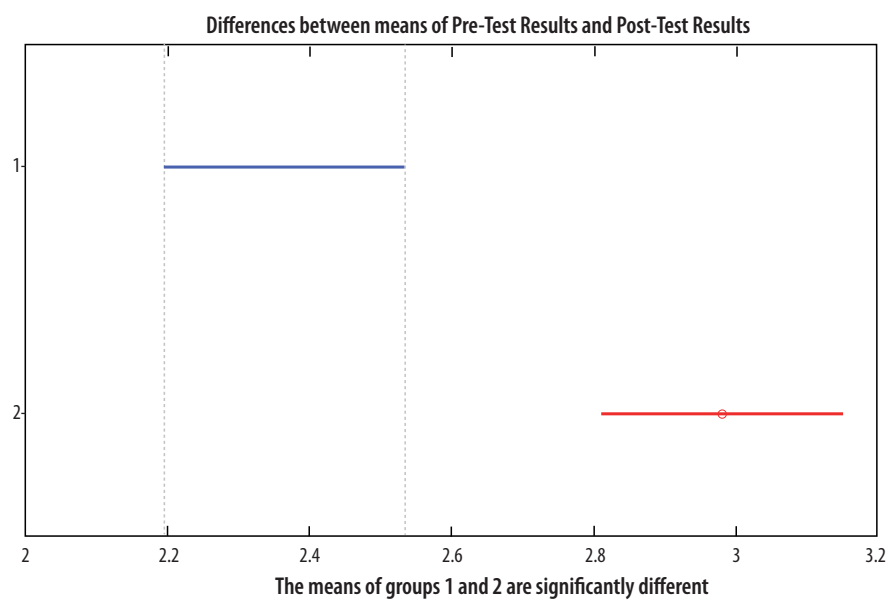

Pretest described in blue and post-test in red. The results of the test show that the post-test sample has a significantly higher mean than the pretest

Source: Prepared by the authors 


\section{Subjective Perceptions Assessment}

The main topics we wanted to assess were personal satisfaction and motivation. On the one hand, we wanted to evaluate if the users were satisfied with the tool during the experience, and, on the other, we wanted to see if their motivation increased after the AR experience.

Regarding the level of satisfaction of the students, 18 out of 20 students (90\%) found the AR development satisfactory and pleasant (between Extremely Satisfied and Very Satisfied). The remaining two students did not find it as pleasant as desired (Moderately Satisfied). These results are shown in Figure 5.

Figure 5. Students' responses regarding their satisfaction with the Augmented Reality Application

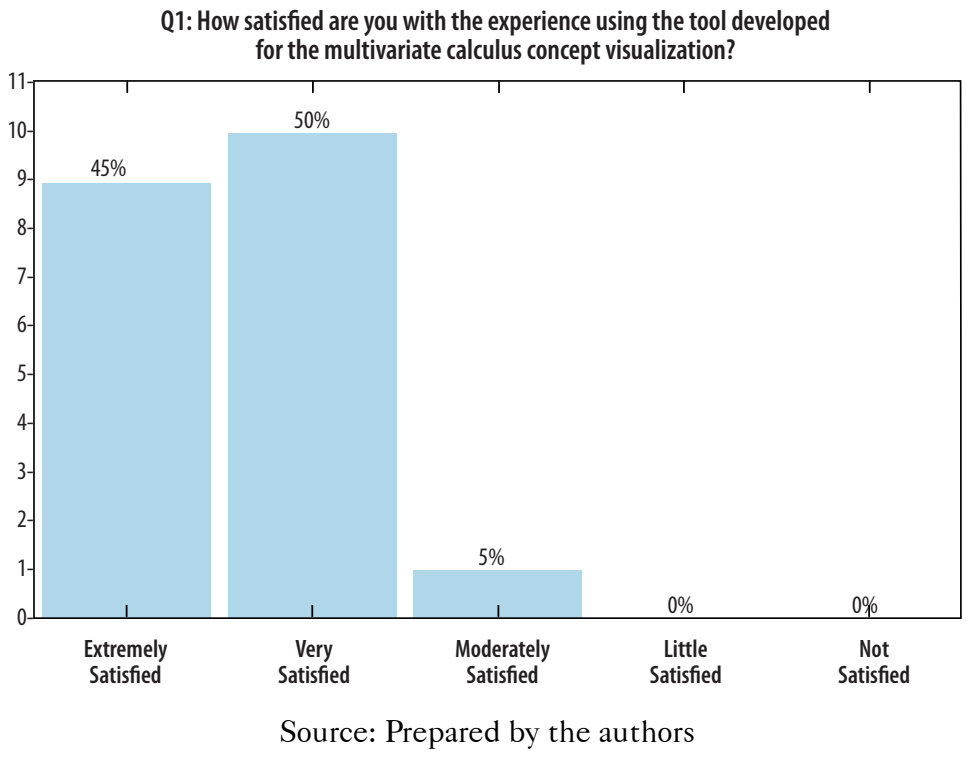

Regarding the motivation of the students in using tools for learning multivariate calculus, 19 out of 20 (95\%) considered that their motivation increased after using the AR tool during the experience and only one student remained neutral, as shown in Figure 6. 
Figure 6. Students' responses on the change in interest in multivariable calculus after the intervention

Q6: How much did your motivation in multivariate calculus learning increased after the use of the tool developed?

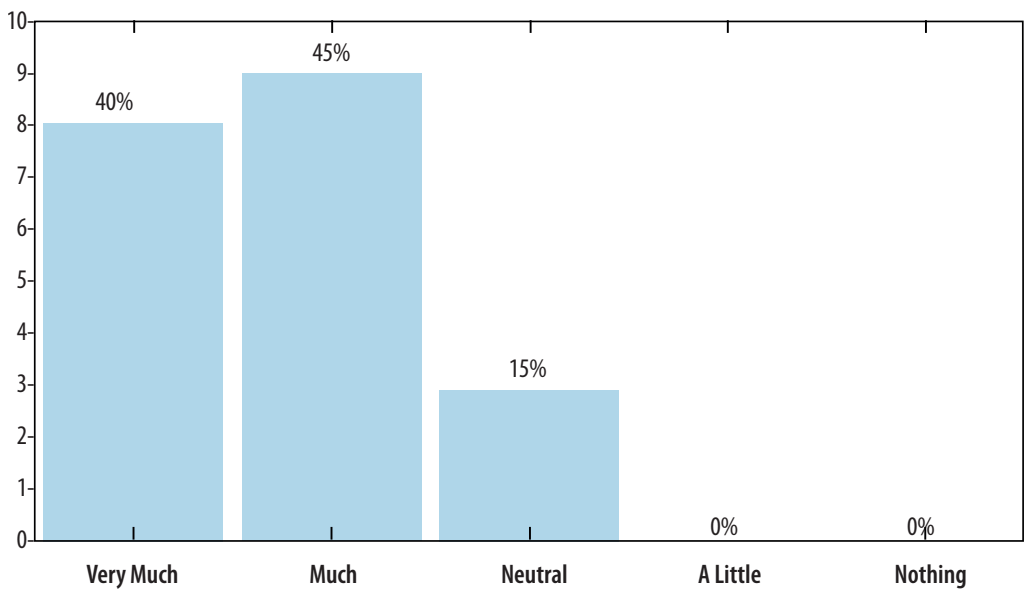

Source: Prepared by the authors

\section{Conclusions and Future Work}

The results of the cognitive assessment suggest that the collaborative AR application created for this project is a valuable tool that can be used in the classroom to support the instructor when introducing the intuitive knowledge underlying various multivariable calculus concepts to students who have not been exposed previously to the concepts. It is important to note that it is not the technology by itself, but the technology handin-hand with appropriate pedagogical guidance through the experience, inviting students to reflect about what they see in the virtual scene, that shows the potential to foster understanding in the students.

We created a very similar VR application to be used with headmounted displays in the classroom. The AR and VR applications were used with students taking a multivariable calculus course; their pedagogical efficacy was tested, and the results were very encouraging. These results go beyond the scope of this chapter and are reported in another publication. 
We are creating additional VR/AR applications to explore their use for teaching physics, with the concepts underlying parabolic motion. It will allow students to perform collaborative laboratory assignments, even if they do not share the same physical location. We also plan to design stand-alone VR/AR applications where an intelligent avatar acts as the instructor, guiding the student through a set of activities and asking them to reflect through smart self-evaluation.

\section{References}

Alhalabi, W. (2016). Virtual reality systems enhance students' achievements in engineering education. Behaviour and Information Technology, 35(11), 919-925. https://doi.org/10.1080/0144929X.2016.1212931

Azuma, R., Baillot, Y., Behringer, R., Feiner, S., Julier, S., \& MacIntyre, B. (2001). Recent advances in augmented reality. IEEE Computer Graphics and Applications, 21(6), 34-47. https://doi.org/10.1109/38.963459

Burdea, G., \& Coiffet, P. (2003). Virtual reality technology. Wiley-IEEE Press.

Chen, P., Liu, X., Cheng, W., \& Huang, R. (2017). A review of using Augmented Reality in Education from 2011 to 2016. In E. Popescu, Kinshuk, M. K. Khribi, R. Huang, M. Jemni, N.-S, C., \& D. Sampson (Eds.), Innovations in smart learning (pp. 13-18). Springer.

https://doi.org/10.1007/978-981-10-2419-1_2

Chudnoff, E. (2013). Intuitive knowledge. Philosophical Studies, 162(2), 359-378. https://doi.org/10.1007/s11098-011-9770-x

Di Serio, Á., Ibáñez, M. B., \& Kloos, C. D. (2013). Impact of an augmented reality system on students' motivation for a visual art course. Computers and Education, 68, 586-596. https://doi.org/10.1016/j.compedu.2012.03.002

Freina, L., \& Ott, M. (2015, April). A literature review on immersive virtual reality in education: state of the art and perspectives [Conference Paper]. In Proceedings of the International Scientific Conference eLearning and Software for Education, Bucarest (pp. 133-141). https://doi.org/10.12753/2066-026x-15-020

Giraldo, F., Rojas, Á. M. J., Esteban, P., \& Trefftz, H. (2007). Collaborative virtual environments for teaching physics. In M. Iskander (Ed.), Innovations in E-Learning, Instruction Technology, Assessment, and Engineering Education (pp. 89-93). https://doi.org/10.1007/978-1-4020-6262-9_16 
GlobeNewswire. (May, 2020). The global augmented reality (AR) and virtual reality (VR) market. Retrieved from https://www.globenewswire. com/news-release/2020/05/29/2041108/0/en/Global-Augmented-Realityand-Virtual-Reality-Market-is-expected-to-reach-USD-161-1-billion-by2025-witnessing-48-8-CAGR-during-the-forecast-period-2020-2025-VynZResearch.html

Holzapfel, B. (2018). The class of 2030 and life-ready learning: The technology imperative. https://info.microsoft.com/ww-landing-McKinsey-Class-Of-2030Whitepaper.html

Ibáñez, M.-B., \& Delgado-Kloos, C. (2018). Augmented reality for STEM learning: A systematic review. Computers and Education, 123, 109-123. https://doi.org/10.1016/j.compedu.2018.05.002

Ibáñez, M.-B., Uriarte Portillo, A., Zatarain Cabada, R., \& Barrón, M. L. (2020). Impact of augmented reality technology on academic achievement and motivation of students from public and private Mexican schools. A case study in a middle-school geometry course. Computers and Education, 145. https://doi.org/10.1016/j.compedu.2019.103734

Manyika, J., Lund, S., Chui, M., Bughin, J., Woetzel, J., Batra, P., Ko, R., \& Sanghvi, S. (2017). Jobs lost, jobs gained: What the future of work will mean for jobs, skills, and wages. McKinsey \& Company. https://www.mckinsey.com/featuredinsights/future-of-work/jobs-lost-jobs-gained-what-the-future-of-work-willmean-for-jobs-skills-and-wages

Jensen, L., \& Konradsen, F. (2018). A review of the use of virtual reality head-mounted displays in education and training. Education and Information Technologies, 23(4), 1515-1529. https://doi.org/10.1007/s10639-017-9676-0

Martín-Gutiérrez, J., Fabiani, P., Benesova, W., Meneses, M. D., \& Mora, C. E. (2015). Augmented reality to promote collaborative and autonomous learning in higher education. Computers in Human Behavior, 51, 752-761. https://doi.org/10.1016/j.chb.2014.11.093

Orozco, C., Esteban, P., \& Trefftz, H. (2006, January). Collaborative and distributed augmented reality in teaching multi-variate calculus [Conference Paper]. In Proceedings of The Fifth IASTED International Conference on Web-Based Education, Puerto Vallarta. ACTA Press. 
Pan, Z., Cheok, A. D., Yang, H., Zhu, J., \& Shi, J. (2006). Virtual reality and mixed reality for virtual learning environments. Computers and Graphics, 30(1), 20-28. https://doi.org/10.1016/j.cag.2005.10.004

Rickel, J. (2001, September). Intelligent virtual agents for education and training: Opportunities and challenges [Conference Paper]. In Proceedings of the Third International Workshop on Intelligent Virtual Agents, Madrid (pp 15-22). Springer. https://doi.org/10.1007/3-540-44812-8_2

Sherin, B. (2006). Common sense clarified: The role of intuitive knowledge in physics problem solving. Journal of Research in Science Teaching, 43(6), 535-555. https://doi.org/10.1002/tea.20136

van den Berk, M. (2015). How Institutions for Higher Education Can Reach New Heights in the Digital Age. ITElligence, 1-19.

Velev, D., \& Zlateva, P. (2017). Virtual Reality Challenges in Education and Training. International Journal of Learning and Teaching, 3(1), 33-37.

https://doi.org/10.18178/ijlt.3.1.33-37

Volkow, S. W., \& Howland, A. C. (2018). The Case for Mixed Reality to Improve Performance. Performance Improvement, 57(4), 29-37.

https://doi.org/10.1002/pfi.21777 